\title{
ESTADO NUTRICIONAL DE PREESCOLARES \\ DE LA COMUNA ALTO BIOBIO Y SU RELACIÓN CON \\ CARACTERÍSTICAS ETNODEMOGRÁFICAS
}

\section{NUTRITIONAL STATUS OF PRE-SCHOOL CHILDREN \\ OF ALTO BIOBIO BOUROUGH AND ITS CORRELATION WITH ETHNO DEMOGRAPHIC FEATURES}

\author{
Claudia Navarrete B. (1), Ricardo Cartes V. (2)
}

(1) CESFAM Ralco, Alto Biobio, Concepción, Chile.

(2) CESFAM Ralco, Servicio de Salud Biobio, Alto Biobio. Concepción, Chile.

\begin{abstract}
Aim: To describe the nutritional status of preschool children of Alto Bio Bio from year 2007 to 2009. Methods: Retrospective descriptive study of the universe of preschools children who were nutritionally evaluated. Data was obtained from the "Healthy Child Control" anthropometric parameters W/H and H/A according to WHO reference and ethno demographic variables were determined. Results: 1,144 preschools were evaluated which were $65.7 \%$ pehuenches and $52.3 \%$ women. Obesity prevalence was $8.15 \%, 8.66 \%$ and $17.17 \%$ in pehuenches, mestizos and westerns respectively. There was a greater risk for malnutrition in mestizos followed by pehuenches. Obesity was higher in boys (10.6\%) and weight deficit was higher in girls (5.3\%). Stunting was higher in men (25.3\%). Conclusions: Coinciding with previous studies we found a tendency to short stature and malnutrition in pehuenches and to obesity in westerns, outcomes associated with extreme poverty in the community.
\end{abstract}

Key words: Pre-school children, ethnic, nutrition, obesity, height.

Este trabajo fue recibido el 10 de Noviembre de 2009 y aceptado para ser publicado el 20 de Diciembre de 2010.

\section{INTRODUCCIÓN}

Los cambios sociales, económicos, políticos, tecnológicos, entre otros han modificado de manera fundamental la forma en que nacemos, crecemos, nos relacionamos, vivimos y morimos, cuestión que se hace claramente patente en la inversión de la pirámide demográfica y en los problemas nutricionales que han afectado al mundo en las últimas décadas. De especial importancia es este último hecho en niños y adolescentes en quienes la prevalencia de sobrepeso y obesidad ha aumentado en una proporción alarmante a nivel mundial, durante las dos últimas décadas, con una prevalencia de sobrepeso y obesidad en niños que alcanza el $20 \%$ en muchos países europeos $(1,2)$.

En Chile, la situación no es diferente y se pasó en menos de 20 años de un estado de pre-transición, con predominio de las enfermedades por déficit, principalmente la desnutrición, a una de post-transición con predominio de obesidad (3). La desnutrición prácticamente ha desaparecido; donde los últimos datos señalan valores menores a $1 \%$ en los menores de seis años (4), sin embargo, aún persiste en grupos de mayor pobreza, especialmente en zonas rurales y donde habita la población indígena (5) los cuales podrían tener una alimentación deficiente, además de otros problemas desencadenados por el círculo de la pobreza (6).

Una variable antropométrica de interés es la estatura, la que ha sido reconocida como un buen indicador de nivel de vida, observándose que los países que presentan un menor desarrollo económico son los que exhiben una mayor prevalencia de talla baja en el niño (7). Se ha señalado que dentro de un mismo país, independientemente de su nivel de desarrollo global, existen variaciones en las frecuencias de déficit de crecimiento, las que estarían relacionadas con los niveles de pobreza local (8-10). Un grupo de interés son los niños indígenas de las zonas de 
alta vulnerabilidad social, quienes al ingresar a la escuela miden $6 \mathrm{~cm}$ menos que el patrón internacional, mientras que los que han migrado a comunas de buen nivel social de Santiago prácticamente tienen una estatura similar a la mediana de esta referencia (11), lo que sugiere un efecto ambiental en la etiología del retraso de crecimiento.

A pesar de existir estudios nacionales que relacionan etnia y estado nutricional, ellos se han centrado en la población mapuche, no existiendo información especifica sobre la población pehuenche, la que habita mayoritariamente en la comuna de Alto Biobio. El objetivo del presente trabajo fue determinar el estado nutricional por antropometría y su relación con las características demográficas y étnicas en un grupo etareo de especial interés del punto de vista sanitario como son los preescolares.

\section{SUJETOS Y MÉTODOS}

El presente trabajo consistió en un estudio de diseño descriptivo, de corte retrospectivo y su universo lo componen los preescolares, 2 a 5 años, atendidos entre los años 2007 y 2009 en la red de salud de la comuna de Alto Biobio, la que se encuentra formada por el CESFAM Ralco, 8 postas y 1 estación médico rural.

El universo estuvo constituido por preescolares con sus controles de acuerdo a la edad $(\mathrm{N}=1144)$. Se excluyeron a $\mathrm{RN}$ pretérmino y a los inasistentes a controles durante los años analizados. Los datos de género, peso, talla y edad fueron extraídos del tarjetón de control de salud del programa del niño en cada uno de los controles a que asistió el preescolar en el consultorio y postas rurales, desde donde se obtuvieron los diagnósticos de estado nutricional y las variables etnodemográficas.

Para los criterios de diagnóstico nutricional fueron utilizados los indicadores peso para la talla $(\mathrm{P} / \mathrm{T})$ y talla para la edad (T/E), recomendados por el Ministerio de Salud con la referencia OMS.

En cuanto a la etnia se clasificaron a los preescolares en 3 grupos: pehuenche, mestizo y occidental. Dicha clasificación se realizó en base a los siguientes criterios:

a. Se consideró pehuenche a aquellos niños con sus dos apellidos indígenas, al igual que sus padres,

b. Mestizos aquellos que solo tenían un apellido pehuenche,

c. Occidentales sin apellidos indígenas.

La información de aquellos apellidos pehuenches fue confirmada por la CONADI y por los Facilitares Interculturales de Salud (paramédicos pehuenches) que trabajan en cada una de las comunidades a las cuales pertenecen los preescolares.

La tabulación de los datos se realizó en el software MS Excel 2003 y el análisis descriptivo en el programa estadístico STATA 10/SE. Dado que se trabajó con el universo de la población en estudio no se realizó estudio del $\mathrm{N}$ muestral ni prueba de significación estadística.

\section{RESULTADOS}

El número total de preescolares incluidos en este estudio fue de 1.144 niñas y niños evaluados entre los años 2007 y 2009 ; de ellos 546 eran varones $(47,7 \%)$ y $598(52,3 \%)$ mujeres. Según la etnia se distribuyeron en pehuenches, mestizos y occidentales representando 752 $(65,7 \%), 206(18,1 \%)$ y $186(16,2 \%)$ respectivamente.

En el gráfico 1 se aprecia que los preescolares de descendencia occidental presentan un mayor porcentaje de malnutrición por exceso $(46,5 \%)$, siendo los pehuenches los que presentan menor prevalencia por esta causa $(30,5 \%)$, tendencia que se mantuvo en los años. La prevalencia de obesidad fue $17,2 \%$ en la población occidental pehuenche de $8,1 \%$ y $8,7 \%$ en mestiza, con una disminución generalizada, pero más notoria en occidentales que bajó de $28 \%$ a 8,3\%, en el período estudiado.

Cabe destacar que la población preescolar de descendencia occidental presentó menos déficit de peso a lo largo de los años, siendo los mestizos quienes mostraron los mayores valores en este aspecto; así el año 2007 fue $4,7 \%$, incrementando a $8,9 \%$ el 2008 y disminuyendo a 5,9\% el año 2009.

En el gráfico 2 se observa que la mayor prevalencia por mal nutrición por exceso según edad fue el año 2007 a los 2 años con un $41,7 \%$, el 2008 a los 3 años con $40,4 \%$ y el año 2009 a los 4 años con $39,4 \%$, lo que se traduce en que un mismo grupo (niños nacidos el 2005) han mantenido su tendencia a la malnutrición por exceso, pero disminuyendo la obesidad a medida que crecen de $18,7 \%$ a $10,6 \%$.

Se aprecia en el gráfico 3 que la tendencia a malnutrición por exceso esta dada mayoritariamente en el sexo masculino para cada uno de los años analizados siendo en promedio $35,7 \%$ en comparación a las mujeres con un $31,3 \%$. También se aprecia que los hombres son los que presentan menores valores para estado nutricional de riesgo de desnutrir en promedio, para los años analizados, lo que representa un $1,3 \%$ en relación a una diferencia importante para sexo femenino, siendo esta de un $5,3 \%$.

El gráfico 4 muestra que los hombres son los que presentan mayor déficit de crecimiento en todos los años analizados con un promedio de $25,3 \%$, siendo para las mujeres $18,8 \%$. Los preescolares que alcanzan mayor porcentaje de normalidad en la talla son de sexo femenino para todos los años analizados lo que representa un $73,6 \%$ en comparación a un $69,2 \%$ para el sexo masculino.

Del gráfico 5 podemos apreciar que a menor edad 
los preescolares tienen una mayor tendencia a presentar déficit en su crecimiento lo que disminuye en edades posteriores. Al observar el gráfico 6 vemos que el grupo de preescolares pehuenches presentan una tendencia a tallas más bajas en relación al grupo de mestizos y en especial a los niños occidentales.

\section{DISCUSIÓN}

Este estudio analizó el estado nutricional de los niños preescolares de la comuna de Alto Biobio, representada mayoritariamente por población de origen pehuenche y con altos niveles de pobreza y extrema pobreza según la última encuesta CASEN (12). Se utili-

\section{GRÁFICO 1}

\section{Distribución de diagnósticos nutricionales según etnia y período}

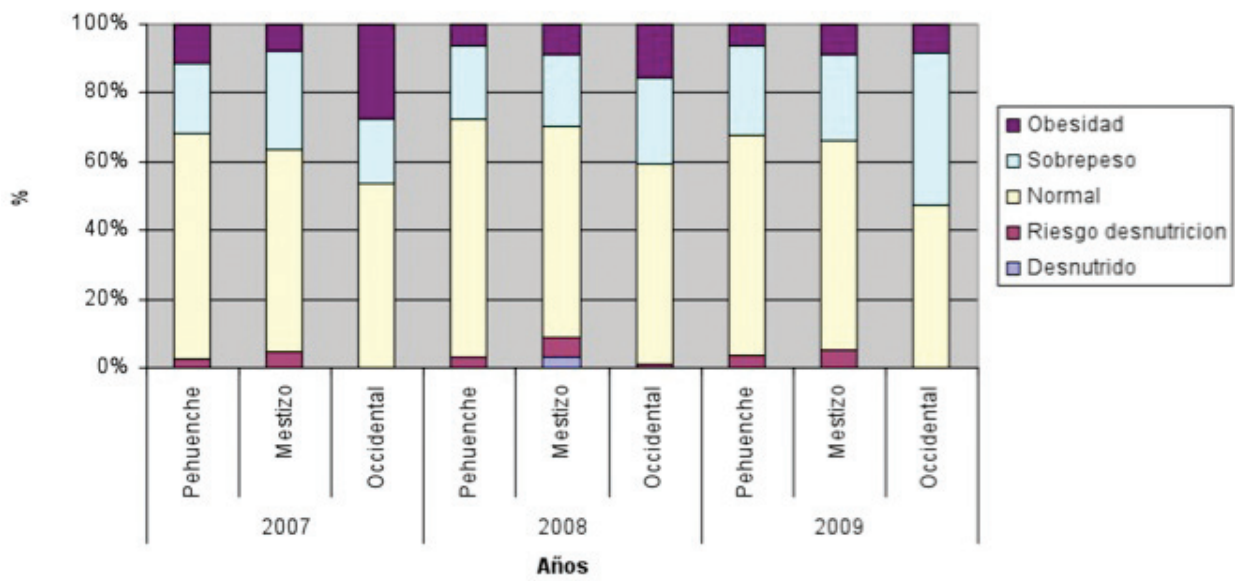

\section{GRÁFICO 2}

Distribución de diagnósticos nutricionales según edad y período

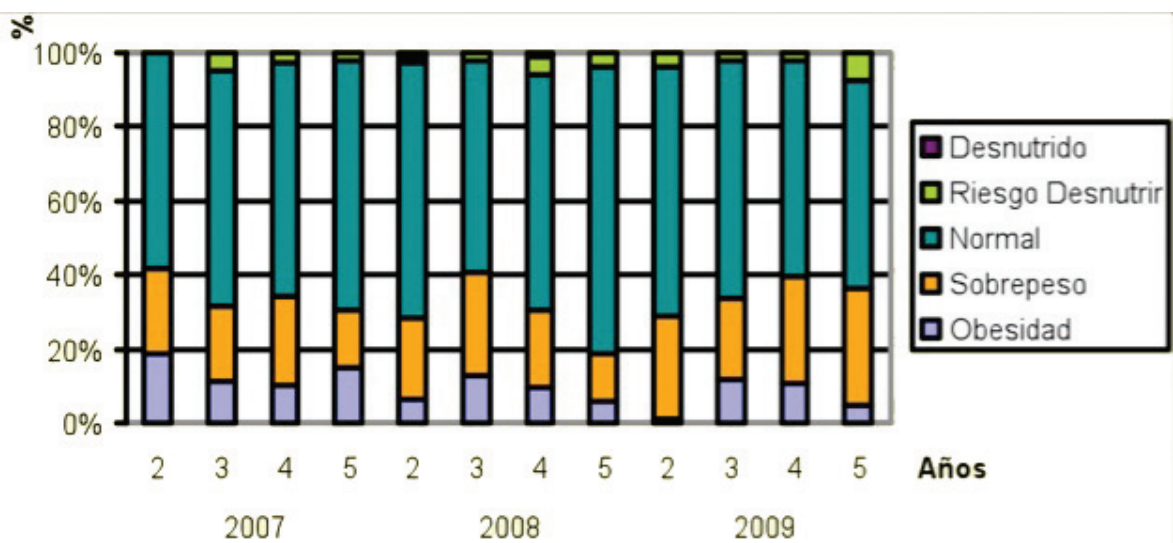


zaron los nuevos estándares OMS, recomendados por el MINSAL a partir del año 2007, los cuales han mostrado cambios en los diagnósticos nutricionales (13), pero que hacen estos resultados comparables con recientes y futuros estudios de esta índole.

Los resultados presentados están en línea con la evidencia nacional e internacional para poblaciones indígenas y socioeconomicamente vulnerables $(5-11,14$, 15), mostrando una clara tendencia hacia la malnutrición por déficit más que por exceso.

Así, encontramos que la población de origen pehuenche es la que presenta una menor proporción de

\section{GRÁFICO 3}

\section{Distribución de diagnósticos nutricionales según sexo y período}

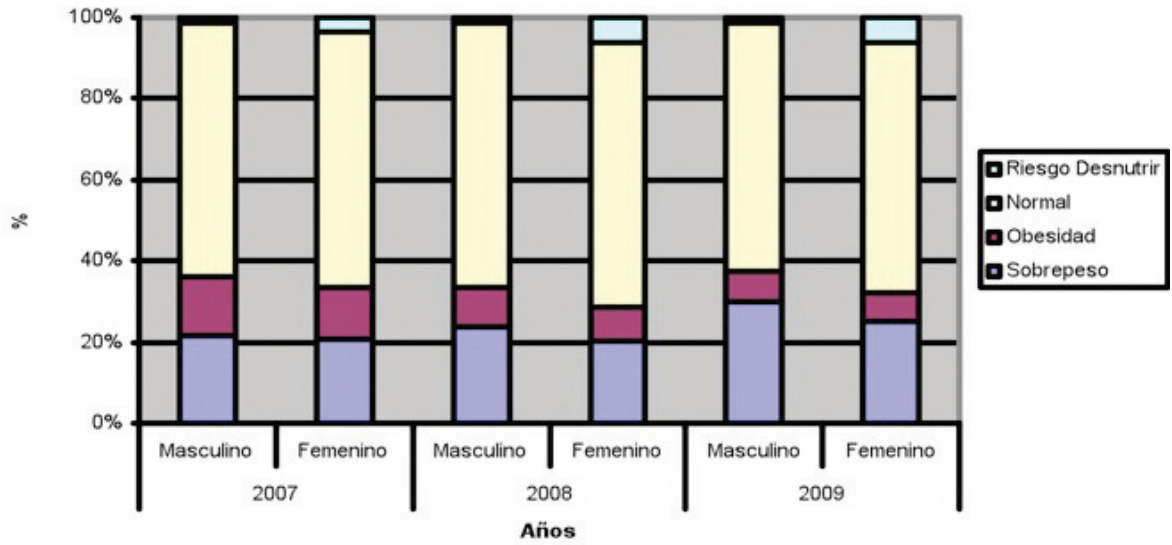

\section{GRÁFICO 4}

Distribución de diagnósticos de crecimiento según sexo y período

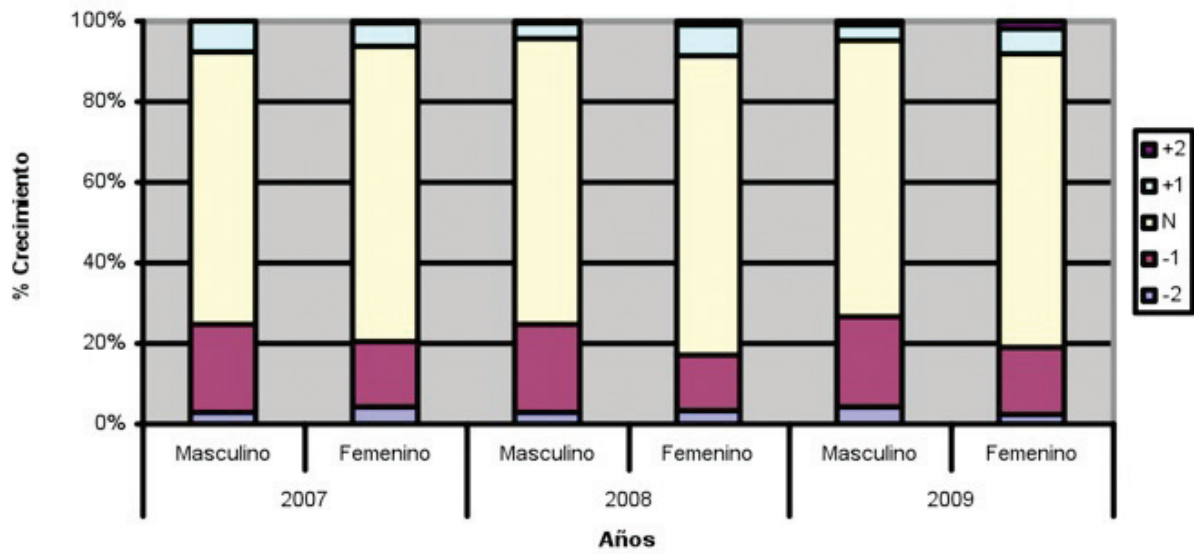


sobrepeso u obesidad y mayores porcentajes de riesgo o desnutrición declarada, esto último comparado con datos nacionales (4). Un hecho de interés es que la población mestiza presenta la mayor desnutrición, lo que se podría deber a que la población occidental (padres o madres de estos preescolares) que llega a la comuna presenta niveles socioeconómicos tan o más bajos que los locales, con lo que se potenciaría su influencia sobre el estado nutricional de sus hijos. Este hecho abriría una línea de investigación ya que ha sido bastante estudiado cuál es la influencia ambiental que se da en indígenas que migran a las ciudades (11), pero no en la situación inversa para

\section{GRÁFICO 5}

Distribución de diagnósticos de crecimiento según edad y período
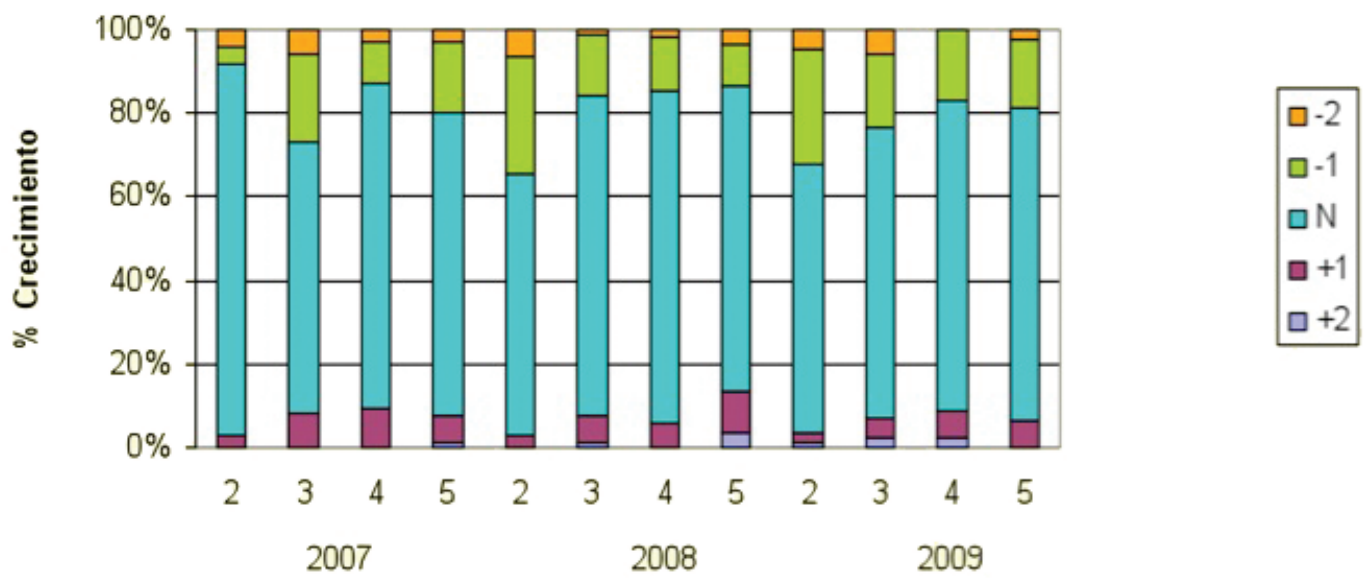

Años

\section{GRÁFICO 6}

Distribución de diagnóstico de crecimiento según etnia y período

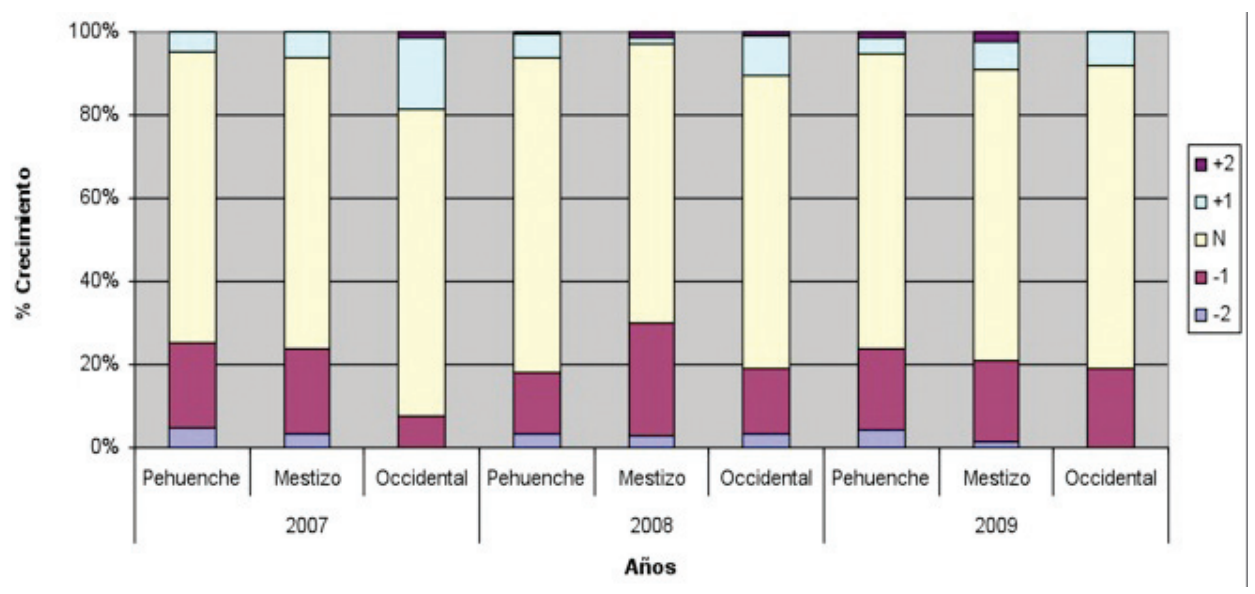


occidentales que por diversas razones migran a localidades rurales e indígenas, nuestros resultados reforzarían la preeminente influencia ambiental (socioeconómica principalmente) sobre el estado nutricional.

En cuanto a los niveles de obesidad estos se concentran preferentemente en población occidental, lo que se debería a que cuentan con mayores recursos económicos para acceder a alimentos hipercalóricos, y que contradice la evidencia que relaciona pobreza con la preferencia por estos alimentos. Esta diferencia se explicaría porque en términos generales la población de nuestra comuna presenta niveles socioeconómicos muy bajos donde los más pobres de este grupo (pehuenches preferentemente) ni siquiera pueden acceder a este tipo de alimentos o lo hacen a una cantidad inferior, a diferencia de la población menos pobre (occidentales en su mayoría) que si lo pueden hacer; lo anterior evidenciaría que en poblaciones de un nivel general socioeconómico bajo o muy bajo la pobreza tiene una influencia inversa sobre los niveles de sobrepeso y obesidad. Se debe hacer un fuerte trabajo educativo y promocional a este respecto para que la población pobre utilice sus pocos recursos económicos en la adquisición de alimentos más sanos.

En relación a la estatura existió una situación similar a lo ya descrito con un mayor déficit en la población indígena si se compara con la occidental; destaca que a medida que los niños crecen esta situación deficitaria tiende a valores normales lo que necesita ser estudiado para ver qué factores influenciarían este hecho y de que forma se pueden intervenir de forma positiva.

En relación al género encontramos que los hombres tienen una tendencia mayor al sobrepeso y a baja estatura en comparación a las mujeres, utilizando las curvas OMS, lo que no tendría una explicación por lo que surge la duda si estas curvas en términos de género son aplicables para esta población o deben pasar por algún tipo de normalización con las consideraciones socioeconómicas, situación que merece ser estudiada porque es importante conocer las referencias de crecimiento ya que facilitan la detección de niños en riesgo de estar desnutridos o sobre nutridos ya que la tendencia se observa mayoritariamente en población indígena a consecuencia de una desnutrición en etapas tempranas de la vida. La información entregada a través de estos estudios refuerza la importancia de focalizar programas de alimentación y desnutrición en estas poblaciones de niños indígenas.

Siendo la comuna más pobre del país, Alto Biobio necesita desarrollar estrategias que impidan que la situación de extrema pobreza siga perjudicando a sus habitantes y especialmente a sus niños y niñas.

\section{LIMITACIONES DEL ESTUDIO}

Existen 2 limitantes para evaluar los resultados presentados en este estudio. Primero las mediciones no fueron tomadas con los fines de esta investigación (estudio retrospectivo basado en registros clínicos), pero todas ellas fueron realizadas por profesionales sanitarios. Segundo: no se pudo incluir el nivel socioeconómico de cada preescolar por no existir una individualización para todas las familias, por lo que no podemos hacer una relación directa, lo que no impide hacer una generalización a partir de los resultados de la encuesta CASEN y la realidad que a diario vemos en la comuna.

\section{CONCLUSIÓN}

Coincidente con los hallazgos de estudios previos, en relación a malnutrición y pueblos indígenas, encontramos que en Alto Biobio la población preescolar pehuenche presenta características de desnutrición y baja estatura, en contraste la occidental presenta una talla normal y mayor tendencia a la malnutrición por exceso. Lo anterior se encuentra asociado a los niveles de extrema pobreza que presenta la comuna.

El presente estudio plantea y refuerza dudas en la investigación nutricional tanto local como nacional, en relación a la influencia de los factores ambientales (socioeconómicos, étnicos, educacionales y de ruralidad) y la necesidad de contar con curvas de crecimiento diferenciadas por etnia.

\section{RESUMEN}

Objetivo: Describir el estado nutricional de preescolares de la comuna Alto Biobio durante el período 20072009. Metodología: Estudio retrospectivo descriptivo del universo de preescolares evaluados nutricionalmente. La información se obtuvo del tarjetón de control de niño sano. Se determinaron los parámetros antropométricos $\mathrm{P} / \mathrm{T}$ y T/E según referencia OMS y variables etnodemográficas. Resultados: Fueron evaluados 1.144 preescolares, $65,7 \%$ correspondían a pehuenches y $52,3 \%$ eran mujeres. La prevalencia de obesidad fue $8,15 \%, 8,66 \%$ y $17,17 \%$ en pehuenches, mestizos y occidentales respectivamente; teniendo mayor riesgo de desnutrición en la etnia mestiza seguida por la pehuenche. La obesidad fue mayor $(10,6 \%)$ en varones, y el déficit de peso fue mayor en las niñas $(5,3 \%)$. La talla baja fue mayor en los varones $(25,3 \%)$. Conclusión: Coincidentemente con estudios previos, encontramos una mayor tendencia a la desnutrición con talla baja en pehuenches y a obesidad con talla normal en occidentales, resultados asociados a la extrema pobreza de la comuna.

Palabras clave: Preescolares, etnia, nutrición, obesidad, estatura. 
Dirigir la correspondencia a:

Profesora

Claudia Navarrete B.

Centro de Salud Familiar Ralco

Calle Domingo Salvo s/n, Ralco

Alto Bio-Bío

CESFAM Ralco, Alto Biobio,

Concepción, Chile

E-mail:claudianavarreteb@yahoo.es

\section{BIBLIOGRAFÍA}

1. Ebbeling CB, Pawlak DB, Ludwig DS. Childhood obesity: public-health crisis, common sense cure. Lancet 2002; 10:473-482.

2. Koletzko B, Girardet JP, Klish W, Tabacco O. Obesity in children and adolescent worldwide: current views and future directions. J Pediatr Gastroenterology Nutr 2002; 35:S205-S212.

3. Vio Fernando, Albala Cecilia: Transición Nutricional en Chile. Rev Chil Nutr. 1998; 25, (3), 11- 22.

4. Albala C, Vio F. Epidemiological transition in Latin America: the case of Chile. Public Health 1995; 109: 431-42

5. Ministerio de Planificación de Chile (MIDEPLAN). Pobreza y distribución del ingreso en Chile 19901998. Santiago, Chile 1999.

6. Psacharopoulos G \& Patrinos H. Los pueblos indígenas y la pobreza en América Latina: Un análisis empírico. Washington, D. C, World Bank 1994

7. UNICEF. The state of the world's children. New
York. New York, UNICEF 2003.

8. Larrea C, Freire W. Social inequality and child malnutrition in four Andean countries. Pan Am J Public Health 2002; 11: 356-64.

9. Amigo H, Bustos P, Radrigan ME, Ureta E. Estado nutricional en escolares de nivel socioeconómico opuesto. Rev Méd Chil. 1995; 123: 1063-70.

10. Hernandez Diaz S, Peterson KE, Dixit S, Hernandez B, Parra S, Barquera S et al. Association of maternal short stature with stunting in Mexican children: common genes v/s common environment. Eur J Clin Nutr 1999; 53: 938-45.

11. Amigo H, Bustos P, Erazo M, Radrigán ME. Comparación del estado nutricional entre escolares indígenas y no indígenas. Rev Med Chil. 1999; 127: 903-10.

12. MIDEPLAN. Encuesta de Caracterización Socioeconómica 2009. Santiago, 2010.

13. González MA, Pino JL. Estudio comparativo de las curvas de crecimiento NCHS/OMS: Evaluación del estado nutricional e implicancias en un centro de salud familiar. Rev Chil Nutr. 2010; 37(2): 169-77.

14. Bustos P, Weitzman M, Amigo H. Crecimiento en talla de niños indígenas y no indígenas chilenos. ALAN 2004; 54(2): 190-5.

15. Bustos P, Muñoz S, Vargas C, Amigo H. Pobreza y procedencia indígena como factores de riesgo en problemas nutricionales de los niños que ingresan a la escuela. Salud Pública Mex. 2009; 51: 187-93. 